\title{
ON REPRESENTING INTEGERS AS PRODUCTS OF INTEGERS OF A PRESCRIBED TYPE
}

\author{
P. D. T. A. ELLIOTT \\ (Received 25 May 1982) \\ Communicated by A. J. van der Poorten
}

\begin{abstract}
A general group-theoretic procedure is indicated for representing rational integers as products of other integers. A detailed example is given.
\end{abstract}

1980 Mathematics subject classification (Amer. Math. Soc.): 10 K 20, 10 L 10, 10 M 99, 12 C 99.

THEOREM. Let

$$
R(x)=\prod_{i=1}^{h}\left(x+a_{i}\right)^{b_{i}}
$$

be a rational function with integer roots $-a_{i} \leqslant 0$, and non-zero exponents whose highest common factor $\left(b_{1}, \ldots, b_{h}\right)$ is 1 . Let an integer $k \geqslant 3$ be given.

Then every positive integer $n$ has a representation of the form

$$
n=\prod_{j} R\left(n_{j}\right)^{\varepsilon_{j}}
$$

where each $\varepsilon_{j}= \pm 1$, and the $n_{j}$ lie in an interval $k \leqslant n_{j} \leqslant c_{0} n$ for some constant $c_{0}$.

The condition $\left(b_{1}, \ldots, b_{h}\right)=1$ is necessary. If, for example, every $b_{i}$ were even, then products of the $R(m)$ could only represent squares of integers.

We cannot at present give an algorithm to determine the constant $c_{0}$.

This theorem illustrates a procedure which may be attempted whenever it is desired to represent one or many integers as products.

Let $Q_{1}$ denote the group of positive rational fractions with multiplication as group law. Let $\Gamma(k)$ denote the subgroup generated by the positive $R(m)$. We

(C) 1983 Australian Mathematical Society $0263-6115 / 83 \$ A 2.00+0.00$ 
form the quotient group $G=Q_{1} / \Gamma(k)$ and construct a proof in three stages by showing that

(i) $G$ is finitely generated,

(ii) $G$ has bounded order,

(iii) $G$ is trivial.

Steps (i) and (ii) are carried out by considering the homomorphisms of $G$ into the additive group of rational numbers ( $\bmod 1)$, and of the reals, respectively.

If both (i) and (ii) have been obtained then $G$ is finite. We have preserved the above formulation since one can sometimes obtain (ii) without (i), and the method may still proceed. Moreover, our following arguments generalise almost at once to a wide class of modules, and an analogue of (ii) can be readily formulated.

Step (iii) applies the homomorphisms of $G$ into the finite fields which have a prime number of elements. In other problems one could use a function field over a finite field, since these permit (non-archimedean) topologies. In order to obtain an appropriate action on $G$ it is convenient to work with the quotients $G / G^{p}$ where $G^{p}$ is the subgroup of $G$ whose elements are $p$ th-powers.

Taken together these three steps form an analogue of the Hardy-Littlewood (circle) method traditionally employed in the additive representation of integers. Our steps (ii) and (iii) correspond to the introduction of the singular integral and singular series of that method, and represent some form of the Hasse "local to global" principle. Step (i) corresponds to the application of algebraic geometry to the study of exponential sums on the so-called minor arcs. For an up-to-date presentation of the classical and some modern applications of the HardyLittlewood method see Vaughan (1981). For an example which illustrates the need for auxiliary information from algebraic geometry see Davenport's paper (1963) on cubic forms.

Product representations of the above type play a rôle in the theory of characters. If a Dirichlet character $\chi()$ satisfies

$$
\chi(R(n))=1 \text { for } k<n \leqslant H
$$

then our theorem shows that

$$
\chi(n)=1 \text { for } 1 \leqslant n \leqslant H / c_{0} .
$$

For non-principal characters this puts a limit on the size $H$ may be. In this way we can obtain a small integer $n$ for which $\chi(R(n))$ is not zero or 1. For background results on character sums see Burgess (1962). For an example of an application of this type (with a different function in the rôle of $R(n)$ ) see Burgess (1967).

Our present method reduces the problem to the consideration of additive arithmetic functions on sequences of various integers. Whilst the study of such 
functions lies within the scope of probabilistic number theory (see, for example, Elliott $(1979 / 80)$ ), in many circumstances only partial results are readily available. Some connections between the present method and others used in obtaining related results, together with an example involving shifted prime numbers, are discussed following the proof of the theorem.

\section{Divisible modules}

Let $G$ be an abelian group (written additively) which becomes a module under the action of a principal ideal domain $R$. We write this action on the left side, thus $r g$ is defined for $r$ in $R$ and $g$ in $G$.

We say that $G$ is a divisible ( $R$-) module if for every element $g$ in $G$ and every non-zero member $r$ of $R$ we can find a further element $h$ in $G$ so that $g=r h$.

LeMma 1. Let $H$ be a submodule of the R-module $G$. Then any homomorphism of $H$ into a divisible $R$-module $D$ can be extended to a homomorphism of $G$ into $D$.

REMARK. In this and what follows the homomorphisms are module homomorphisms.

Proof. When $R$ is $\mathbf{Z}$, the ring of rational integers, this result appears as exercise 1 in Kaplansky's book (1969) on infinite abelian groups.

We consider the collection of all pairs $(K, t)$ of submodules $K$ containing $H$ which have a homomorphism $t$ into $D$ extending that defined on $H$. We partially order these pairs by

$$
(K, t) \succ\left(K^{\prime}, t^{\prime}\right)
$$

if $K$ includes $K^{\prime}$ and $t$ extends $t^{\prime}$. It is readily checked that any chain has an upper bound, and by Zorn's lemma the collection contains a maximal pair, $(L, \tau)$ say.

Suppose that $L$ is not $G$, and let $g$ be an element of $G$ not in $L$. Let $\Delta$ be the submodule generated by $g$ and $L$.

If $m g$ does not lie in $L$ for any non-zero member $m$ of $R$ then $\Delta$ is the direct sum of $L$ and the module generated by $g$. We may define a map $T: \Delta \rightarrow D$ by

$$
T(m g+\lambda)=\tau(\lambda)
$$

for all $\lambda$ in $L$.

Otherwise there will be a non-trivial ideal of elements $m$ in $R$ so that $m g$ lies in $L$. Since $R$ is principal this ideal will be generated by an element, $\pi$ say. 
We now appeal to the divisibility of the module $D$, and let $\delta$ be an element of it for which $\tau(\pi g)=\pi \delta$. We then define

$$
T(m g+\lambda)=m \delta+\tau(\lambda) .
$$

Note that if $m_{1} g+\lambda_{1}=m_{2} g+\lambda_{2}$ then $\left(m_{1}-m_{2}\right) g$ belongs to $L$, so that in $R$ $m_{1}-m_{2}$ must be a multiple of $\pi$, say $k \pi$. Hence

$$
\begin{aligned}
T\left(m_{1} g+\lambda_{1}\right)-T\left(m_{2} g+\lambda_{2}\right) & =\left(m_{1}-m_{2}\right) \delta+\tau\left(\lambda_{1}\right)-\tau\left(\lambda_{2}\right) \\
& =k \pi \delta+\tau\left(\lambda_{1}-\lambda_{2}\right)=k \pi \delta+\tau\left(\left\{m_{2}-m_{1}\right\} g\right) \\
& =k \pi \delta-k \tau(\pi g)=0,
\end{aligned}
$$

so that $T$ is well defined.

In either case we obtain a genuine extension

$$
(\Delta, T)>(L, \tau)
$$

contradicting the maximality of $(L, \tau)$.

Thus $L=G$ and the lemma is proved.

We say that a homomorphism is trivial on a set of elements of a module if it takes each of them to zero (the identity).

In what follows $D$ will be a divisible module containing at least two elements.

Lemma 2. Let $G_{1}$ and $G_{2}$ be submodules of an $R$-module $G_{1}$. If every homomorphism of $G$ into $D$ which is trivial on $G_{1}$ is also trivial on $G_{2}$, then to each element $g$ in $G_{2}$ there is a non-zero member $r$ of $R$ so that $r g$ lies in $G_{1}$.

Proof. Suppose, to the contrary, that no product $r g$ with $r$ in $R$ of the element $g$ of $G_{2}$ lies in $G_{1}$. Let $\Delta$ be the module generated by $g$ and $G_{1}$. Since $\Delta$ is the direct sum of $G_{1}$ and the module generated by $g$, we may define a homomorphism $t$ of $\Delta$ into $D$ by setting $t(g)$ to be any non-zero element in $D$, and defining

$$
t(r g+\mu)=r t(g)
$$

for each element $\mu$ of $G_{1}$.

By Lemma $1 t$ may be extended to a homomorphism of $G$ into $D$. Moreover, this new homomorphism is trivial on $G_{1}$ but not on $G_{2}$, contradicting the hypothesis of the lemma.

Lemma 2 is established.

ReMARK. In our applications it will not be assumed that the modules $G_{1}$ and $G_{2}$ have any non-trivial intersection.

Any abelian group may be considered a $\mathbf{Z}$-module. An abelian group which is divisible as a Z-module we shall call a divisible abelian group without mentioning 
its module structure. In this case we can reformulate Lemma 2 as

LEMMA 3. Let $G_{1}$ and $G_{2}$ be subgroups of an abelian group $G$. Suppose that every homomorphism of $G$ into a (non-trivial) divisible group $D$ which is trivial on $G_{1}$, is also trivial on $G_{2}$. Then every element in $G_{2}$ has a positive multiple in $G_{1}$.

REMARK. In our applications of this lemma it will be convenient to take for $D$ the additive group of the real numbers, which is clearly divisible.

For groups which have torsion better can sometimes be done. Let $p$ be a rational number prime number and let $G$ be a possibly infinite abelian group, each of whose non-trivial elements has order $p$. Let $F_{p}$ be a finite field of $p$ elements.

We can make $F_{p}$ act on $G$ by identifying $F_{p}$ with the field of integer residue classes $(\bmod p), \mathbf{Z} / p \mathbf{Z}$, and using the rule

$$
(n(\bmod p), g) \mapsto n g .
$$

In view of the $p$-torsion this action is well defined. $G$ now becomes a vector space over $F_{p}$.

The analogue of Lemma 3 is now

LEMMA 4. Let $G_{1}$ and $G_{2}$ be subgroups of an abelian group $G$ with p-torsion. Suppose that every homomorphism of $G$ into a non-trivial vector space over $F_{p}$ which is trivial on $G_{1}$ is also trivial on $G_{2}$. Then $G_{2}$ is contained in $G_{1}$.

Proof. Since $F_{p}$ is a field, any vector space over $F_{p}$ is $F_{p}$-divisible. According to Lemma 2 with $R=F_{p}$, to each element $g$ of $G_{2}$ there is a non-zero member $r$ of $F_{p}$ so that $r g$ belongs to $G_{1}$. Once again using that $F_{p}$ is a field, there is a member $s$ of $F_{p}$ so that $s r=1$, and therefore $g=s(r g)$ itself belongs to $G_{1}$.

REMARK. In our application of Lemma 4 groups $G$ arise which need not have $p$-torsion; so we give them it by considering the factor group $G / G^{p}$, where $G^{p}$ denotes the subgroup of $p$ th-powers of elements in $G$.

AN EXAMPLE. Let $q$ be a rational prime and let $p_{1}<p_{2}<\cdots$ run through all the rational primes. Define the integers

$$
a_{1}=p_{1}^{q^{2}}, \quad a_{j+1}=\left(p_{1} \cdots p_{j+1}\right)^{q}\left(p_{1} \cdots p_{j}\right)^{-1}, \quad j=1,2, \ldots
$$

Let $A$ be the subgroup $Q_{1}$ which is generated by these $a_{i}$. 
If for any $i \geqslant 2$ and integer $m p_{i}^{m}$ belongs to $A$, then there will be a representation

$$
p_{i}^{m}=\prod_{l=1}^{s} a_{l}^{d_{l}}
$$

for integers $d_{l}$, and $s \geqslant 1$. Since each $p_{j+1}$ occurs in $a_{j+1}$ and in no $a_{w}$ with $w \leqslant j$, we see that $s \leqslant i$ must hold. Then $m=q d_{i}$.

On the other hand (group theoretically)

$$
p_{j+1}^{q} \equiv\left(p_{1} \cdots p_{j}\right)^{q-1} \quad(\bmod A),
$$

and an easy inductive proof shows that

$$
p_{i}^{q^{i+1}} \equiv 1 \quad(\bmod A), \quad i=1,2, \ldots
$$

We see that every element of the group $Q_{1} / A$ has an order which is a power of $q$, and which is at least $q$.

Since every element of $Q_{1} / A$ has a finite order all homomorphisms of it into the additive group of real numbers are trivial. Likewise it cannot have a non-trivial homomorphism into an $F_{p}$ with $p \neq q$. Moreover,

$$
p_{j}=\frac{p_{2} \cdots p_{j}}{p_{2} \cdots p_{j-1}} \equiv \frac{\left(p_{1} \cdots p_{j+1}\right)^{q}}{\left(p_{1} \cdots p_{j}\right)^{q}} \equiv p_{j+1}^{q} \quad(\bmod A)
$$

for $j \geqslant 2$, and

$$
p_{1}=\frac{p_{1} p_{2}}{p_{2}} \equiv \frac{\left(p_{1} p_{2} p_{3}\right)^{q}}{p_{2}} \quad(\bmod A)
$$

so that every element of $Q_{1} / A$ is the $q$ th-power. Thus it has no non-trivial homomorphisms into $F_{q}$.

Since no $p_{i}$ belongs to $A$ it is clear that the vanishing of the homomorphisms into the additive group of the reals, together with those into the finite fields $F_{p}$ is not enough to ensure the triviality of $Q_{1} / A$, and therefore the representation of integers as products of the $a_{j}$.

The reason for this is that the homomorphisms have ranges in groups which do not (necessarily) possess enough structure. By considering maps into more structured groups better may be done.

Let $D_{1}$ be a divisible $R$-module with an identity. Suppose that for each prime element $\pi$ of $R$ there is a non-zero element $g$ of $D_{1}$ so that $\pi g_{1}=0$.

Lemma 5. Let $G_{1}$ and $G_{2}$ be sub-modules of an $R$-module G. Suppose that every homomorphism of $G$ into $D_{1}$ which is trivial on $G_{1}$ is also trivial on $G_{2}$. Then $G_{2}$ is contained in $G_{1}$. 
Proof. For each $g$ in $G_{2}$, Lemma 2 guarantees that the ideal of elements $r$ in $R$ for which $r g$ belongs to $G_{1}$ is not empty.

Suppose that for some $g$ in $G_{2}$ this ideal is non-trivial, and is generated by $\alpha$. Let $\pi$ be a prime element of $R$ which divides $\alpha$ and set $y u=\pi^{-1} \alpha g$. Then $y$ belongs to $G_{2}$ but not to $G_{1}$. Moreover, $\pi y$ lies in $G_{1}$.

Let $\Delta$ be the module generated by $G_{1}$ and $y$. We define a map $T$ of $\Delta$ into $D_{1}$ by choosing a non-zero element $\delta$ of $D_{1}$ which satisfies $\pi \delta=0$ and setting

$$
T(r y+\mu)=r \delta
$$

for every $r$ in $R$ and $\mu$ in $G_{1}$. If $r_{1} y+\mu_{1}=r_{2} y+\mu_{2}$ then $\left(r_{1}-r_{2}\right) y$ belongs to $G_{1}$, so that $\pi$ divides $r_{1}-r_{2}$. Let $r_{1}-r_{2}=\rho \pi$. Then

$$
T\left(r_{1} y+\mu_{1}\right)-T\left(r_{2} y+\mu_{2}\right)=\left(r_{1}-r_{2}\right) \delta=\rho \pi \delta=0,
$$

and $T$ is well defined.

By Lemma 1 we may extend $T$ to a homomorphism of $G$ into $D_{1}$, which is then trivial on $G_{1}$ but not $G_{2}$. This contradicts the hypothesis of the lemma.

Lemma 5 is proved.

A candidate for $D_{1}$ is the multiplicative group of complex numbers which are roots of unity, or its isomorphic copy the additive group $Q / \mathbf{Z}$ of rationals $(\bmod 1)$.

\section{A ring of operators}

Let $S$ be an $R$-module, containing at least two elements, defined over an integral domain $R$ which has an identity. Consider the set of all doubly-infinite sequences $\left(\ldots, s_{-1}, s_{0}, s_{1}, s_{2}, \ldots\right)$ of elements of $s$. We introduce the shift operator $E$ whose action takes a typical sequence $\left\{s_{n}\right\}$ to the new sequence $\left\{s_{n+1}\right\}$. If $F(x)=\sum_{j=1}^{r} c_{j} x^{j}$ is a polynomial with coefficients in $K$, we extend this definition by defining

$$
F(E) s_{n}=\sum_{j=1}^{r} c_{j} s_{n+j}
$$

In this way we define a ring of operators which is isomorphic to the ring of polynomials with coefficients in $K$. In what follows operator will mean a (polynomial) operator which belongs to this ring.

Let $K$ be the quotient ring of $R$.

LEMMa 6. Let $F(x)$ be a polynomial in $R[x]$ which factorises into

$$
a \sum_{i=1}^{r}\left(x-\theta_{i}\right)
$$


over some extension field of $K$. Then for each positive integer $d$

$$
a^{r d} \sum_{i=1}^{r}\left(x-\theta_{i}^{d}\right)
$$

also belongs to $R[x]$.

If, furthermore, $R$ is integrally closed, then the polynomial

$$
a^{r d} \prod_{i=1}^{r}\left(x^{d}-\theta_{i}^{d}\right)
$$

is divisible by $F(x)$ in $R[x]$.

Remark. For the properties of integral closure see Zariski and Samuel (1962) Chapter V.

Proof. Consider the polynomial

$$
\prod_{i=1}^{r}\left(x-y_{i}^{d}\right)
$$

with the $y_{i}$ distinct indeterminates over $K$. The coefficients $b_{j}$ of $s^{j}, 0<j<r$, is a symmetric function of the $y_{i}$, of total degree $(r-j) d$. If $\sigma_{v}, \nu=0, \ldots, r$, denotes the elementary symmetric functions of the $y_{i}$, then $b_{j}$ is a polynomial in these $\sigma_{\nu}$, of degree at most $r d$. (See, for example, van der Waerden (1953) Volume 1, Chapter 26.)

Specialising the $y_{i}$ to $\theta_{i}$, we see from our first hypothesis that every $a \sigma_{\nu}$ belongs to $R$. Hence $a^{r d} b_{j}$ belongs to $R$ for every $j$, which justifies the first assertion of the lemma.

Consider next the polynomial

$$
W(x)=a^{r d} \prod_{i=1}^{r}\left(s^{d}-\theta_{i}^{d}\right) .
$$

Clearly each factor $x^{d}-\theta_{i}^{d}$ is divisible by $x-\theta_{i}$ in some algebraic extension of $K$. By working in a large enough extension $F(x)$ will divide $W(x)$. Since $K$ is a field $F(x)$ then divides $W(x)$ in $K[x]$.

For each root $\theta_{i}$ of $F(x)=0, a \theta_{i}$ is integral over $R$. The coefficients of the polynomial

$$
a^{d-1} \frac{x^{d}-\theta_{i}^{d}}{x-\theta_{i}}
$$

are thus integral over $R$, and so are those of the polynomial $W(x) R(x)^{-1}$.

Since $R$ is integrally closed in its quotient field, this last polynomial actually belongs to $R[x]$.

The lemma is proved. 
In our next two lemmas and in their application, $R$ will be a unique factorisation integral domain with identity.

A function $f(n)$ is said to be arithmetic if it is defined on the positive natural integers. We shall say that it is additive if it takes values in $S$ and satisfies the relation

$$
f(a b)=f(a)+f(b)
$$

for all positive integers $a$ and $b$. In the theory of numbers one traditionally requires this relation only to hold if $a$ and $b$ have no common factor other than 1 . We shall not need this limitation. Thus our additive arithmetic functions are restrictions, to the integers, of homomorphisms of the group of positive rational fractions.

We extend the sequence $f(1), f(2), \ldots$, of values of an arithmetic function to a doubly infinite sequence by setting $f(n)=0$ if $n \leqslant 0$.

Note that if $f()$ is an arithmetic function

$$
E f(2 n)=f(2 n+1) .
$$

If, however, we define a new arithmetic function $g()$ by $g(n)=f(2 n)$ then

$$
E g(n)=g(n+1)=f(2 n+2) .
$$

LEMMA 7. In the above notation suppose that the additive arithmetic function $f()$ satisfies

$$
\psi(E) f(n)=\text { constant }, \quad k \leqslant n \leqslant H,
$$

for some operator $\psi(E)$. Let

$$
\psi(x)=a \sum_{i=1}^{s}\left(x-\omega_{i}\right)^{r_{i}},
$$

with distinct $\omega_{i}$, hold over some extension field of $K$. Let $t=r_{1}+\cdots+r_{s}$ denote the degree of $\psi(x)$. Let a positive integer $d$ be given.

Then either there is a permutation $\sigma$ of the $\omega_{i}$ with

$$
\sigma\left(\omega_{i}\right)=\omega_{i}^{d}, \quad i=1, \ldots, s,
$$

or there is a further non-zero polynomial $\psi_{1}(x)$, defined over $R$ and with degree less than that of $\psi(x)$, so that

$$
\psi_{1}(E) f(n)=(\text { another }) \text { constant }
$$

holds over the interval $k \leqslant n \leqslant(H / d)-t$.

Proof. Consider the polynomial $G(x)=a^{t d} \prod_{i=1}^{s}\left(x-\omega_{i}^{d}\right)^{r_{i}}$. By Lemma $6 \psi(x)$ divides $G\left(x^{d}\right)$ in $R[x]$. Therefore

$$
G\left(E^{d}\right) f(n)=\text { constant }
$$

for $k \leqslant n \leqslant H-m_{1}$ where $m_{1}=\operatorname{deg}\left(G\left(x^{d}\right) / \psi(x)\right) \leqslant t(d-1)$. 
Let $G(x)=\Sigma_{j=0}^{\prime} c_{j} x^{j}$. Then $G\left(E^{d}\right) f(n)=\Sigma_{j=0}^{t} c_{j} f(n+d j)$, so that for $k \leqslant n d$ $\leqslant H-m_{1}$

$$
\begin{aligned}
\sum_{j=0}^{t} c_{j} f(n+j) & =\sum_{j=0}^{t} c_{j}[f(d\{n+j\})-f(d)] \\
& =G\left(E^{d}\right) f(n d)-G(1) f(d)=\text { constant. }
\end{aligned}
$$

In particular

$$
G(E) f(n)=\text { constant }
$$

over the range $k \leqslant n \leqslant(H / d)-t$.

If the roots of $G$ are a permutation of the $\omega_{i}$ (in both cases neglecting the multiplicities $r_{i}$ ) we obtain the first of the two possibilities appearing in the statement of Lemma 7. Otherwise $G(x)$ and $a^{t d-1} \psi(x)$ have the same leading terms, but are distinct. With $\psi_{1}(x)=a^{t d-1} \psi(x)-G(x)$ we then have the second of the possibilities.

Lemma 7 is proved.

LEMMA 8. Let

$$
\psi(E) f(n)=\text { constant }, \quad k \leqslant n \leqslant H,
$$

where $\psi(x)$ is a polynomial over $R$ of degree $t$. Let $d$ be an integer, $d \geqslant 2$.

Then there are integers $q, 0 \leqslant q \leqslant t$, and a non-zero element $\delta$ of $R$, such that

$$
\delta(E-1)^{q} f(n)=0 \text { for } k+t \leqslant n \leqslant H d^{-t^{2}(t+1)}-2 t .
$$

Moreover, if $H \geqslant 2^{11 t^{3}} k^{2}$ and $S$ is a field, then

$$
f(n)=0 \quad \text { for } k+t \leqslant n \leqslant 2^{-6 t^{3}} H .
$$

REMARK. The same value of $\delta$ may serve for all the $H$ which satisfy the hypothesis of the lemma.

Proof. The hypotheses of Lemma 7 are satisfied. Suppose that a permutation $\sigma$ with the property (2) of that lemma exists. Consider a cycle in the permutation, say,

$$
z_{1} \rightarrow z_{2} \rightarrow \cdots \rightarrow z_{h} \rightarrow z_{1}
$$

so that $z_{j}=\sigma z_{j-1}, j=1, \ldots, h$. Then by (2)

$$
z_{1}=\sigma z_{h}=z_{h}^{d}=\left(\sigma z_{h-1}\right)^{d}=\cdots=z_{1}^{d^{h}},
$$

giving $z_{1}^{d^{h}-1}=1$. In this way every $\operatorname{root} \omega_{i}$ of $\psi(x)$ is seen to be a root of unity, $\omega_{i}^{d_{i}}=1$ say, and each $d_{i}$ is a divisor of one of the numbers $d^{w}-1,1 \leqslant w \leqslant s$. 
Let

$$
D=\prod_{w=1}^{s}\left(d^{w}-1\right)<d^{s^{2}} \leqslant d^{t^{2}} .
$$

Then $\omega_{i}^{D}=1$ for every $i$. Clearly $\psi(x)$ divides the polynomial

$$
a^{i D} \prod_{i=1}^{s}\left(x^{D}-\omega_{i}^{D}\right)^{r_{i}}
$$

giving

$$
a^{t D}\left(E^{D}-1\right)^{t} f(n)=\text { constant }
$$

over the interval $k \leqslant n \leqslant H-t(D-1)$. Arguing as in the proof of Lemma 7 we replace $n$ by $D n$ and reach

$$
a^{i D}(E-1)^{t} f(n)=\mathrm{constant}
$$

for $k \leqslant n<(H / D)-t$.

It is convenient at this point to consider the alternative (3) presented in Lemma 7. This has the form of the hypothesis of the present lemma save that the degree of $\psi_{1}(x)$ is less than that of $\psi(x)$, and the range $[k, H]$ is reduced to $[k,(H / D)-t]$.

Assume that $t \geqslant 1$. We may argue inductively to reach an integer $q, 0 \leqslant q \leqslant t$, a non-zero element $\delta$, such that

$$
\delta(E-1)^{q} f(n)=\text { constant }
$$

holds for

$$
k+t \leqslant n \leqslant \frac{H}{d^{t^{2}(t-q+1)}}-t\left(1+\frac{1}{d^{t^{2}}}+\cdots+\frac{1}{d^{t^{2}(t-q)}}\right),
$$

and certainly over the range $k \leqslant n \leqslant H d^{-t^{2}(t+1)}-2 t$.

If now $S$ is a field, and $q \geqslant 1$, we set $s(n)=(E-1)^{q-1} f(n)$ and over this same range have $s(n+1)-s(n)=c_{0}$, say, giving $s(n)=c_{0} n+c_{1}$ for certain constants $c_{0}, c_{1}$. Proceeding inductively in this manner we obtain a polynomial $g(y)$, of degree at most $t$, such that

$$
f(n)=g(n) \text { for } k+t \leqslant n \leqslant H d^{-t^{2}(t+1)}-2 t .
$$

We next note that so long as $k^{2} \leqslant n^{2} \leqslant H d^{-t^{2}(t+1)}-2 t$ we have

$$
g\left(n^{2}\right)-2 g(n)=f\left(n^{2}\right)-2 f(n)=0 .
$$

If now $H>2 t(4 t+k)^{2} d^{t^{2}(t+1)}, t \geqslant 1$, and $S$ has characteristic zero, the polynomial $g\left(x^{2}\right)-2 g(x)$, which is of degree at most $2 t$, will have more than $2 t$ distinct (integer) roots. It must therefore be identically zero, that is to say $g(x)$ must be a constant. 
The same argument may still be made unless $S$ is a field of finite characteristic $p \leqslant 2 t$. For such fields we have

$$
f(p)+f(n)=f(p n)=g(0) \text { for } k+t \leqslant p n \leqslant H d^{-t^{2}(t+1)}-2 t .
$$

Thus in every case

$$
f(n)=\text { constant, } \quad k+t \leqslant n \leqslant(2 t)^{-1} H d^{-t^{2}(t+1)}-1 .
$$

Denoting this constant by $c$ we have $2 c=2 f(k+t)=f\left((k+t)^{2}\right)=c$, giving $c=0$.

The proof of the lemma is now completed by setting $d=2$ and treating the simple case $t=0$ directly.

\section{Proof of the theorem}

As in the introduction let $Q_{1}$ denote the multiplicative group of positive rational numbers.

Let $G_{1}$ denote its subgroup generated by the fractions

$$
R(l) \text { with } k \leqslant l \leqslant H,
$$

and let $G_{2}$ be the subgroup generated by the integers in the interval $k+t \leqslant n \leqslant$ $2^{-6 t^{3}} H$.

Let $W$ be the group $G_{2} / G_{1}$, viewed as the subgroup of $Q_{1} / G_{1}$ which is generated by the cosets $g\left(\bmod G_{1}\right)$ as $g$ runs through the elements of $G_{2}$.

Suppose that $f^{*}$ is a homomorphism of $W$ into an $R$-divisible group $\Gamma$, where $R$ is a principal ideal domain which acts upon $W$ and $Q_{1} / G_{1}$. Then by Lemma 1 there is an extension of $f^{*}$ which maps the whole of $Q_{1} / G_{1}$ into $\Gamma$. Thus there is an additive function

$$
f: Q_{\mathbf{l}} \rightarrow \Gamma
$$

which is trivial on the subgroup $G_{1}$, and which is consistent with $f^{*}$ when suitably restricted.

For a homomorphism $f()$ of $Q_{1}$ into $\Gamma$ to be trivial on $G_{1}$ we must have $f(R(l))=0$, that is

$$
\sum_{i=1}^{h} b_{i} f\left(l+a_{i}\right)=0
$$

for each integer $l$ in $[k, H]$. Here we have assumed that the rational integers can be given a suitable interpretation in $R$. 
(i) If now $H \geqslant 2^{11 t^{3}} k^{2}$ and $\Gamma=S$ is the additive group $Q / Z$, regarded as a $\mathrm{Z}$-module, then we may apply Lemma 8 with

$$
\psi(x)=\sum_{i=1}^{h} b_{i} x^{a_{i}}, \quad t=\max a_{i},
$$

to obtain integers $m>0$ and $q, 0 \leqslant q \leqslant t$, so that

$$
m(E-1)^{q} f(n)=0, \quad k+t \leqslant n \leqslant H 2^{-t^{2}(t+1)}-2 t .
$$

Note that $S$ is not a field, so we are not permitted to appeal to the second assertion of that lemma.

However, if $q \geqslant 1$ the function $m(E-1)^{q-1} f(n)$ is constant on the interval $\left[k+t, 2^{-6 t^{3}} H\right]$ and, if $f(j)=0, k+t \leqslant j \leqslant k+2 t$, will be zero there.

Arguing inductively we see that the assumption (4) now forces $m f(n)$ to be zero over the whole range $\left[k, 2^{-6 t^{3}} H\right]$.

In our above notation: if $f^{*}$ is trivial on the subgroup of $W$ generated by the cosets $j\left(\bmod G_{1}\right), j=k, \ldots, k+t$, then $f^{*}$ is trivial on $W^{m}$ the group of $m$ th-powers of the elements in $W$.

By Lemma 5 the group $W^{m}$ is finitely generated, with the $j\left(\bmod G_{1}\right)$ as generators. Moreover, the value of $m$ does not depend upon the value of $H$.

(ii) We now apply the above argument with $\Gamma=S$ the additive group of the real numbers, regarded as a $\mathbf{Z}$-module. In fact we can apply Lemma 2 directly. In this case the hypothesis (4) leads to the conclusion

$$
f(n)=0 \text { on }\left[k+t, 2^{-6 t^{3}} H\right],
$$

for the reals are a field and we may apply the full force of Lemma 8 .

Since each of the above integers $j$ lies in the interval $\left[k+t, 2^{-6 t^{3}} H\right]$, there are positive integers $\mu_{j}$ so that

$$
j^{\mu_{j}} \equiv 1 \quad\left(\bmod G_{1}\right) \text {. }
$$

Let $\mu$ denote their product. Then each $g$ in $W$ satisfies $g^{m} \equiv \prod_{j=k+t}^{k+2 t} j^{s_{j}}\left(\bmod G_{1}\right)$ for some $s_{j}$, and so

$$
g^{m \mu} \equiv 1\left(\bmod G_{1}\right)
$$

Thus $W$ has bounded order.

This brings us to the end of stage (ii) of the proof. We have shown that for each integer $n>k+t$ there is a representation

$$
n^{m \mu}=\prod_{i} R\left(n_{i}\right)^{\varepsilon_{i}}
$$

with $k \leqslant n_{i} \leqslant 4^{6 t^{3}}\left(n+k^{2}\right)$. Moreover, the value of the exponent $m \mu$ does not depend upon $H$. However, we cannot give bounds for the $\mu_{j}$ and so for $\mu$. As we 
tighten our grip upon the exponent of $n$, the constant $c_{0}$ which appears in the statement of the theorem begins to slip away from us.

(iii) To complete our proof we apply this argument with $\Gamma=S=F_{p}$, a finite field of $p$ elements, but with $Q_{1}$ replaced by $Q_{1} / Q_{1}^{p}, G_{j}$ by $G_{j} / Q_{1}^{p}$.

Once again $S$ is a field, and for an $f$ which takes values in $F_{p}$ to vanish on $G_{1} / Q$ p we must have

$$
\psi(E) f(n)=0 \quad \text { for } k \leqslant n \leqslant H .
$$

Here the polynomial $\psi(x)$ is interpreted by considering the coefficients as in the residue class field $\mathbf{Z} / p \mathbf{Z}$. Since as rational integers the $b_{i}$ have highest common factor $1, \psi(x)$ will not then vanish identically.

We conclude from Lemma 8 that

$$
f(n)=0 \text { on }\left[0,2^{-6 t^{3} H}\right] .
$$

Thus $G_{2} \subseteq G_{1} Q_{1}^{p}$ for every prime $p$.

In particular each integer $n \geqslant k+t$ has a representation

$$
n=z^{p} \prod_{j} R\left(r_{j}\right)^{\nu_{j}}
$$

with $v_{j}= \pm 1$ and $k \leqslant r_{j} \leqslant 4^{6 t^{3}}\left(n+k^{2}\right)$. Clearly the primes which appear in a canonical factorization of the fraction $z$ do not exceed

$$
\max _{1 \leqslant i \leqslant h} 4^{6 t^{3}}\left(n+k^{2}+a_{i}\right)<c_{1} n .
$$

If now $m \mu>1$ and $p$ divides $m \mu$, then

$$
n^{p^{-1} m \mu}=z^{m \mu} \prod_{j} R\left(r_{j}\right)^{\nu_{j}}=\prod_{i} R\left(n_{i}\right)^{\varepsilon_{i}}
$$

this time with $k \leqslant n_{i} \leqslant 4^{6 t^{3}}\left(c_{1} n+k^{2}\right)$. Note that if $z$ has a prime factor $t$ which is less than $k$, then we consider it as a ratio $(k+t) s /(k+t)$.

Arguing inductively we strip off the primes in $m \mu$ to reach

$$
n=\prod_{i} R\left(n_{i}\right)^{\varepsilon_{i}}
$$

with $k \leqslant n_{i} \leqslant c_{1}^{0+1}$, where $v$ denotes the total number of prime divisors of $m \mu$. With $c_{0}=c_{1}^{v+1}$ the theorem is proved.

Since we do not have a bound for $v, c_{0}$ cannot be computed. 


\section{Sets of uniqueness}

Let us now adopt the less restrictive condition that an arithmetic function $f()$ be called additive if

$$
f(a b)=f(a)+f(b)
$$

whenever the (positive) integers $a$ and $b$ are coprime, and completely additive if this relation holds for all pairs of integers. The homomorphisms which were applied in the earlier part of this paper were thus defined by completely additive arithmetic functions.

A sequence

$$
A: a_{1}<a_{2}<\cdots
$$

of positive integers with the property that every real additive arithmetic function which vanished on them also vanished identically, was said by Kátai (1968a) to be a set of uniqueness. In particular, he proved (1968b) that if to the sequence

$$
P: 3<4<6<\cdots<p+1<\cdots \text {, }
$$

where the $p$ are primes, we adjoin finitely many integers then we obtain a set of uniqueness. He conjectured that $P$ itself was a set of uniqueness. This was established to be true by the author, Elliott (1974).

It was proved by Wolke (1978) and Dress and Volkmann (1978) that if a sequence $A$ is a set of uniqueness for an additive arithmetic function then every positive integer has a representation

$$
n^{h}=\prod a_{j_{i}}^{\varepsilon\left(j_{i}\right)}
$$

with $\varepsilon\left(j_{i}\right)= \pm 1$. The $h$ may vary with $n$. This amounts to a form of Lemma 3 with the additive group of the real numbers as $D$. Our present method differs in the following regard:

We deal with modules, rather than vector spaces over the rationals as they did, and we localise the integers used in the product representation.

It followed from the author's proof of Kátai's conjecture that (as Wolke, and Dress and Volkmann mentioned) there is a representation

$$
n^{h}=\prod\left(p_{i}+1\right)^{\varepsilon_{i}}
$$

with the $p_{i}$ prime and $\varepsilon_{i}= \pm 1$.

Let

$$
M(x)=\max _{1 \leqslant n \leqslant x}|f(x)|, \quad E(x)=\max _{p \leqslant x}|f(p+1)| .
$$

In a later paper the author (Elliott (1976)) proved that for completely additive functions $f(n)$ there are positive (absolute) constants so that

$$
M(x) \leqslant A E\left(x^{B}\right)
$$


holds for $x \geqslant 2$. In view of our present Lemma 3 this now shows that the primes in the representation (5) may be restricted to the range $p \leqslant n^{B}$. Assuming only that $f()$ be additive I established only the weaker result

$$
M(x) \leqslant A E\left(x^{B}\right)+A M\left((\log x)^{C}\right)
$$

for some $C>0$.

That (6) holds for all additive function $f()$ was proved by Wirsing (1980), and he strengthened the representation (5) by restricting the primes $p_{i}$ to lie in an interval $n<p_{i} \leqslant n^{B}$, and having both $h$ and the total number of factors in the product bounded above independently of $n$.

If $P_{1}$ denotes the subgroup of $Q_{1}$ which is generated by the $\left(p_{i}+1\right)$ in particular Wirsing's result shows that $Q_{1} / P_{1}$ has bounded order. For the sequence $P$ this brings us to the end of stage (ii) of the general procedure discussed at the beginning of the present paper.

In order to prove that every integer $n$ has a representation

$$
n=\prod_{i}\left(p_{i}+1\right)^{\varepsilon_{i}}, \quad \varepsilon_{i}= \pm 1,
$$

it is sufficient (and also necessary) that for each prime $q$, a completely additive arithmetic function $f()$ with values in the integers $(\bmod q)$ which satisfies $f(p+1) \equiv 0(\bmod q)$ for all primes $p$ must also satisfy $f(n) \equiv 0(\bmod q)$ for every positive integer $n$.

\section{Multiplicative functions}

An arithmetic function $\phi(n)$ is said to be multiplicative if it satisfies

$$
\phi(a b)=\phi(a) \phi(b)
$$

for all pairs of positive coprime integers $a, b$ and to be completely multiplicative if this relation holds for all positive integers $a$ and $b$.

We can now state

LEMMA 9. Let $a_{1}, a_{2}, \ldots$, be a sequence of positive integers. In order that every positive integer may have a representation of the form

$$
n=\prod_{j=1}^{s} a_{j}^{d_{j}}
$$

for some integers $d_{j}$, positive negative or zero, it is necessary and sufficient that every completely multiplicative arithmetic function which is 1 on the $a_{j}$ and whose values are roots of unity, be identically 1. 
Proof. We apply Lemma 5 with $G_{2}=G=Q_{1}, G_{1}$, the subgroup of $Q_{1}$ generated by the $a_{n}$, and consider maps into the multiplicative group of roots of unity.

A form of this result would be implicit in Theorem 2 of Dress and Volkmann (1978). There they were interested in what properties a sequence $a_{j}$ must have in order that one could reconstruct a complex-valued completely multiplicative function $\phi()$ from its values $\phi\left(a_{j}\right)$. In particular $\phi(n)$ was allowed to be sometimes zero. However, the proof which they give is not complete.

Translated into our present circumstances, let $V$ be the subgroups of $Q_{1}$ generated by the $a_{j}$ in Lemma 9 . They aim to prove that $Q_{1} / V$ is trivial by showing that otherwise one can construct a complex-valued multiplicative $\phi()$ which is 1 on the $a_{j}$, but not identically 1 .

Employing a form of Lemma 3 (see our earlier comments on their method) they prove that every element of $Q_{1} / V$ has a finite order. By adjoining rational primes to $V$ a larger group $V_{1}$ is obtained so that the order of each element in $Q_{1} / V_{1}$ is divisible by some particular prime $q$. A further group $V_{2}$ is now formed by adjoining to $V_{1}$ the $q$ th-powers of the rational primes needed to generate $Q_{1} / V_{1}$, and (as they maintain) one obtains a group $Q_{1} / V_{2}$ each of whose non-trivial elements has order $q$.

Their aim is now to obtain a non-trivial map of $Q_{1} / V_{1}$ into some vector space over the field $F_{q}$, and for that they need $Q_{1} / V_{2}$ to be non-trivial. This, however, need not be the case. If, for example, $V$ is generated by the integers at (1) then in the above argument $V=V_{1}$, with every element of $Q_{1} / V_{1}$ being a $q$ th-power. The construction of $V_{2}$ by Dress and Volkmann now gives $Q_{1}=V_{2}$.

One may instead argue as follows. Let $G$ be the group $Q_{1} / V$. If for some prime $p$ the group $G / G^{p}$ is non-trivial then (regarded as vector spaces over $F_{p}$ ) there exists a non-trivial homomorphism of $G / G^{p}$ into $\mathrm{C}_{p}$, the multiplicative group of $p$ th roots of unity. We have

$$
Q_{1} \rightarrow Q_{1} / V \rightarrow G / G^{p} \rightarrow \mathbf{C}_{p}
$$

where the first two maps are the natural projections. This defines a non-trivial completely multiplicative function $\phi(n)$ on $Q_{1}$ which has the value 1 on $V$.

Otherwise $G=G^{p}$ for every prime $p$. The group $G$ is thus a torsion group which is divisible. Such groups are the direct sum for varying rational primes $p$, of isomorphic copies of the group $\mathbf{Z}\left(p^{\infty}\right)$ (Kaplansky (1969) Theorem 4). This last is the group of rational numbers $(\bmod 1)$ which are generated by the fractions whose denominators are powers of the prime $p$. In particular $\mathbf{Z}\left(p^{\infty}\right)$ is isomorphic to the multiplicative group $C_{p}^{*}$ of roots of unity whose orders are powers of $p$. This gives

$$
Q_{1} \rightarrow Q_{1} / V \rightarrow \mathbf{Z}\left(p^{\infty}\right) \rightarrow \mathbf{C}_{P}^{*}
$$


where the first map is the natural projection, the second is by means of a projection onto one of the direct summands isomorphic to $\mathbf{Z}\left(p^{\infty}\right)$, and the last is an isomorphism. Once again we obtain a non-trivial multiplicative $\phi(n)$ which is one on $V$.

Since no such $\phi()$ exists $Q_{1} / V$ must be trivial, as asserted.

This method of proof is interesting in that it illustrates how much of the structure of $Q_{1} / V$ is determined by homomorphisms into the additive reals or the finite fields $F_{p}$.

In our proof of the (main) theorem maps into the additive real rather than the multiplicative complex numbers were employed, since addition is generally more familiar than multiplication. The steps (i)-(iii) are practical in other examples.

\section{Simultaneous representation}

Let $Q_{2}=Q_{1} \oplus Q_{1}$ be the direct sum of two copies of the multiplicative group of positive rationals. Let $a_{n}, n=1,2, \ldots$, and $b_{m}, m=1,2, \ldots$, be two infinite sequences of integers, and let $G$ be the subgroup generated by the pairs $a_{n} \oplus b_{n}$.

If $f()$ is a homomorphism of $Q_{2}$ into (for example) the additive group of the real numbers, then we can decompose it as

$$
f()=f_{1}()+f_{2}()
$$

where

$$
f_{1}(r \oplus s)=f(r \oplus 0), \quad f_{2}(r \oplus s)=f(0 \oplus r)
$$

for all $r$ and $s$ in $Q_{1}$. This naturally defines two maps of $Q_{1}$ into $\mathbf{R}$.

The group $Q_{2} / G$ is now studied by considering those additive functions $f_{i}(n)$, $i=1,2$, which satisfy

$$
f_{1}\left(a_{n}\right)+f_{2}\left(b_{n}\right)=0
$$

for all $n$. If these must vanish identically, then to each pair of positive integers $m_{1}$, $m_{2}$ we can find further integers $k>0, n_{i}>0, \varepsilon_{i}= \pm 1, i=1, \ldots, s$, so that (simultaneously)

$$
m_{1}^{k}=\prod_{i=1}^{s} a_{n_{i}}^{\varepsilon_{i}}, \quad m_{2}^{k}=\prod_{i=1}^{s} b_{n_{i}}^{\varepsilon_{i}} .
$$

The preceding theory may thus be adapted to deal with the simultaneous representation of integers. For example, let $\left(m_{1}, 3\right)=1,\left(m_{2}, 5\right)=1$ hold. Then we can obtain the representation

$$
m_{1}^{k}=\prod_{i=1}^{s}\left(3 n_{i}+1\right)^{\varepsilon_{i}}, \quad m_{2}^{k}=\prod_{i=1}^{s}\left(5 n_{i}+2\right)^{\varepsilon_{i}} .
$$


Since the complications increase, however, we shall furnish the details of such an application on another occasion.

\section{References}

D. A. Burgess (1962), 'On character sums and primitive roots', Proc. London Math. Soc. 12, 179-192

D. A. Burgess (1967), 'On the quadratic character of a polynomial', J. London Math. Soc. 42, 73-80.

H. Davenport (1963), 'Cubic forms in 16 variables', Proc. Roy. Soc. Ser. A 272, 285-303.

F. Dress and B. Volkmann, 'Ensembles d'unicité pour les fonctions arithmétiques additives ou multiplicatives', C. R. Acad. Sci. Paris Ser. A 287, 43-46.

P. D. T. A. Elliott (1979/80), Probabilistic number theory I, II (Springer, New York, Heidelberg, Berlin).

P. D. T. A. Elliott (1974), 'A conjecture of Kátai', Acta Arith. 26, 11-20.

P. D. T. A. Elliott (1976), 'On two conjectures of Kátai', Acta Arith. 30, 35-59.

I. Kaplansky (1969), Infinite abelian groups, revised edition (University of Michigan Press, Ann Arbor).

I. Kátai (1968a), 'On sets characterising number-theoretical functions', Acta Arith. 13 315-320.

I. Kátai (1968b), 'On sets characterising number-theoretical functions (II), (The set of "prime plus one"s is a set of quasi-uniqueness)', Acta Arith. 16, 1-4.

R. C. Vaughan (1981), The Hardy Littlewood method (Cambridge University Tract No. 80).

B. van der Waerden (1953), Modern algebra (Ungar, New York, 1931).

E. Wirsing (1980), 'Additive functions with restrictive growth on the numbers of the form $p+1$ ', Acta Arith. 37, 345-357.

D. Wolke (1978), 'Bemerkungen über Eindeutigkeitsmengen additiver Funktionen', Elem. Math. 33, 14-16.

O. Zariski and P. Samuel, Commutative algebra (Van Nostrand, Toronto, London, New York, 1958).

Department of Mathematics

University of Colorado

Boulder, Colorado 80309

U.S.A. 